\section{Important distinction between acute coronary syndromes and Takotsubo syndrome}

\author{
Vincenzo Marafioti(D, Giulia Turri(i) and Salvatore Monaco (1)
}

We thank Waterbury and colleagues for their elegant Review (Non-atherosclerotic causes of acute coronary syndromes. Nat. Rev. Cardiol. https://doi.org/10.1038/s41569-019-0273-3 $(2019))^{1}$ and wish to discuss the allusive and ambiguous term 'acute coronary syndrome' (ACS) that is applied to Takotsubo syndrome (TTS).

In the Review ${ }^{1}$, the authors state that the "typical clinical presentation of TTS mimics that of ACS" but later state that TTS is a "notable [cause] of non-atherosclerotic ACS". The epithet 'non-atherosclerotic' (similarly to the vague neologism 'myocardial infarction with non-obstructed coronary arteries' or MINOCA $^{2}$ ) does not remove the contextual ambiguity. The term 'ACS' is supposed to mean any group of clinical symptoms compatible with acute myocardial ischaemia and includes unstable angina, non-STsegment elevation myocardial infarction and ST-segment elevation myocardial infarction. By contrast, TTS is described by the ACC and AHA as a distinct morphological manifestation of stress cardiomyopathy, characterized by transient left ventricular dysfunction, electrocardiographic changes that can mimic (but are not) those of acute myocardial infarction and minimal release of myocardial enzymes in the absence of an obstructed coronary artery ${ }^{3}$. Therefore, according to this taxonomy, the term ACS is not a synonym for TTS.
The authors of the Review seem to favour a primary role of the coronary arteries in the pathogenesis of TTS, overlooking without question the transient left ventricular apical ballooning and the important effect of the brain on the heart ${ }^{4}$. Of note, the characteristic pattern of ventricular wall-motion abnormality in TTS is congruent with the distribution of cardiac nerves mapped by the anatomist and neurologist Antonio Scarpa ${ }^{5}$. The supposed microvascular spasm might be an epiphenomenon rather than the cause of TTS.

Moreover, the myocardial histological changes that occur in ACS are distinct from those in TTS. In ischaemic myocardial necrosis (that is, in ACS), the cells die in an atonic state, with no myofibrillar damage. Conversely, endomyocardial biopsy samples from some patients with TTS show a different histological pattern, in which the cells die in a hypercontracted state, with early myofibrillar damage and anomalous, irregular cross-band formations ${ }^{6}$. These cardiac lesions, termed myocytolysis ${ }^{7}$, are caused by a direct effect of catecholamines on cardiomyocyte $\beta$-adrenergic receptors and are not primarily mediated by vasoconstriction. These lesion areas are not located around vessels but at the ends of intracardiac nerves, as observed in other conditions that are unrelated to a substantial reduction in coronary blood flow . $^{8}$

In conclusion, by using the terms 'cardiomyopathy of TTS' and 'non-atherosclerotic
ACS' interchangeably, a semantic problem is introduced that might cause confusion to clinicians. We must not forget that disease taxonomy is one of the foundations of medical science and has an important role in defining the International Classification of Diseases. Accordingly, scientists must take great care to use the appropriate nomenclature, because each word is tightly bound to its meaning, and its value depends on its accurate use.

Vincenzo Marafioti (D) ${ }^{*}$, Giulia Turri (D) ${ }^{2}$ and Salvatore Monaco iD ${ }^{2}$

'Cardiovascular and Thoracic Department, University Hospital of Verona, Verona, Italy. ${ }^{2}$ Department of Neurological and Movement Sciences, University Hospital of Verona, Verona, Italy. *e-mail: vincenzo.marafioti@fastwebnet.it https://doi.org/10.1038/s41569-020-0342-7

1. Waterbury, T. M. et al. Non-atherosclerotic causes of acute coronary syndromes. Nat. Rev. Cardiol. https:// doi.org/10.1038/s41569-019-0273-3 (2019).

2. Pasupathy, S., Tavella, R. \& Beltrame, J. F. Myocardial infarction with nonobstructive coronary arteries (MINOCA): the past, present and future management. Circulation 135, 1490-1493 (2017).

3. Maron, B. J. et al. Contemporary definitions and classification of the cardiomyopathies: an American Heart Association scientific statement from the Council on Clinical Cardiology, Heart Failure and Transplantation Committee; Quality of Care and Outcomes Research and Functional Genomics and Translational Biology Interdisciplinary Working Groups; and Council on Epidemiology and Prevention. Circulation 113, 1807-1816 (2006).

4. Marafioti, V., Turri, G., Carbone, V. \& Monaco, S. Association of prolonged OTc interval with Takotsubo cardiomyopathy: a neurocardiac syndrome inside the mystery of the insula of Reil. Clin. Cardiol. 41, 551-555 (2018).

5. Scarpa, A. Tabulae Neurologicae Ad Illustrandam Historiam Anatomicam, Cardiacorum Nervorum, Noni Nervorum Cerebri, Glossopharyngaei, Et Pharyngaei Ex Octavo Cerebri [Latin] (Ticini, Balthasarem Comini, 1794).

6. Nef, H. M. et al. Tako-Tsubo cardiomyopathy: intraindividual structural analysis in the acute phase and after functional recovery. Eur. Heart J. 28, 2456-2464 (2007).

7. Turillazzi, E. et al. A systematic study of a myocardial lesion: colliquative myocytolysis. Int. J. Cardiol. 104 152-157 (2005).

8. Greenhoot, J. H. \& Reichenbach, D. D. Cardiac injury and subarachnoid hemorrhage, a clinical, pathological, and physiological correlation. J. Neurosurg. 30, 521-531 (1969).

\section{Competing interests}

The authors declare no competing interests. 